\title{
Design of a micro device to electrically separate and count $w b c$ from whole blood
}

\author{
Soumya Gangopadhyay ${ }^{1}$,AVimala Juliet ${ }^{2}$ \\ ${ }^{1,2}$ (Department of Instrumentation and ControlEngineering,SRM University, India)
}

\begin{abstract}
This work presents a micro device which separates and counts Leukocytes (white blood cell or WBC) from other two type of blood cells, Erythrocytes (red blood cell or RBC), and Thrombocytes (Platelet). The operation is performed using four basic parts, a micro mixer which mixes whole blood and diluent to dilute the blood, a size dependent electrical cell separator which separates WBC from other blood cells, a micro nozzle to increase the flow velocity of cells, a counter unit to count the number of WBC in sample. The size dependent cell sorting is done by electrical pillar arrays. The focusing of incoming cells to the counter is done by focusing electrodes. The main aim of this work is to count the separated white blood cells and it is done by a MEMS basedCoulter counter. Simple structure and ease of operation is the two main features of this device. The design of the device has been done by simulating different unit of the device in ComsolMultiphysics. Simulation results were satisfactory and each unit is able to perform its work as per requirement.
\end{abstract}

Keywords-Coulter counter, deterministic lateral displacement, Dielectrophoresis (DEP), micro mixer and micro nozzle.

\section{Introduction}

There are efforts on cell separation and counting systems [1], [2], [3], [4]. There are different systems to separate the blood cells e.g. fluorescence spectroscopy, magnetophoretic cell separation, physical pillar based separation etc. But each of these systems have their own problems like fluorescence spectroscopy can't count the cells separately it gives an average count of cells and most importantly it is not possible to fabricate it in a micro device. The magnetophoretic cell separator uses two permanent magnets which is not possible to place in close range of micro device. The physical pillar based cell separator can do size based cell separation and can be fabricated in a micro device, but after some use of the device the cells tend to stick to the channel floor and create clogging [1]. In this work dielectrophoresis has been used for separating the blood cells which would allow the cells neither to settle down to the channel floor nor to slow down during the process. In previous micro Coulter counter devices there was a problem, there was a chance that more than one cells could get lumped together and the counter would have been counting them as one [4]. In this work focusing electrodes has been used for focusing the cell flow so that no two cells can get lumped together and the counter would count them individually. In this work the dilution system has been implemented in the device. Hence no dilution needs to be done before injecting the blood to the device.This device is a combination of the cell separation and counting system which increases the efficiency to count the number of the most important cell in human blood which causes most of the infectious diseases.

The three blood cells in human blood are respectively Erythrocyte (red blood cell or RBC), Leukocyte (white blood cell or WBC) and Thrombocyte (Platelet). TABLE 1 compares the size of the three main blood cells

TABLE 1. Comparison of wbc, rbc and platelet.

\begin{tabular}{|c|c|}
\hline Blood Cells & Cell Diameter $(\mu \mathrm{m})$ \\
\hline WBC & $7.72-15$ \\
\hline RBC & $6-8$ \\
\hline Platelet & $2.65-2.9$ \\
\hline
\end{tabular}

TABLE 2. Description of different wbc.

\begin{tabular}{|l|l|l|}
\hline \multicolumn{1}{|c|}{ Type } & $\begin{array}{c}\text { Approx. \% in } \\
\text { adults }\end{array}$ & Diameter $(\mu \mathrm{m})$ \\
\hline Neutrophil & 62 & $10-12$ \\
\hline Eosinophil & 2.3 & $10-12$ \\
\hline Basophil & 0.4 & $12-15$ \\
\hline Lymphocyte & 30 & $7-15$ \\
\hline Monocyte & 5.3 & $7.72-9.99$ \\
\hline
\end{tabular}


WBC is of five main types neutrophil, eosinophil, basophil, lymphocyte and monocyte. TABLE 2 describes their size and amount of presence in blood among the total leukocytes.

As the size of WBC ranges from 7.72 to $15 \mu \mathrm{m}$, so the cells having lesser diameter than $7.72 \mu \mathrm{m}$ is to be rejected and the greater or equal diameter cell is to be selected and forwarded for the counting purpose.

\subsection{Micro Mixer Unit}

\section{Device Principle}

Blood needs to get diluted before it can be used for any test because if it is not diluted, the test solution becomes over populated by cells and the count can't be precise. Hence the blood is $1^{\text {st }}$ diluted to a certain percentage to reduce the number of cells per unit sample. Another reason to dilute the blood is to prevent clotting of blood in the channel.This unit mixes the blood with PBS (Phospheted Buffer Saline) to dilute the blood.

This unit has a simple structure as shown in figure. 1 in which the blood and PBS is injected into the device from two different inlets and directed to a junction where the two fluid streams get mixed and then moved through a zigzag long channel to give both the fluids to get mixed well and then finally the mixture or the diluted blood is released in a specific point of the cell separator unit.

In case of the fabricated device the dilution factor is to be used is $1: 100$ i.e. $0.1 \mathrm{ml}$ of whole blood is to be mixed with $9.9 \mathrm{ml}$ of PBS to prepare a $10 \mathrm{ml}$ of diluted blood. The naturel count of WBC in blood is 4500 to $10800 / \mu \mathrm{l}$. After dilution the count reduces to 45 to $108 / \mu \mathrm{l}$. It reduces the time to count the cells for the device. Dilution Factor $=\frac{\text { Sample Volume }}{\text { Total Volume }}$

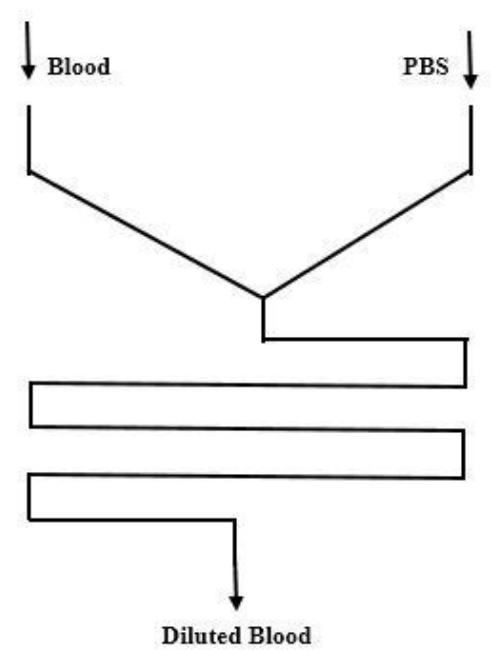

Figure1 Micro mixer unit.

\subsection{Cell Separation Unit}

This unit consists of a two dimensional array of circular spot electrodeswhere each of the row of the array is shifted by $\triangle X$. Each row consists of alternate pairs of $V_{D E P}$ and GND electrodes. DEP force is generated by these electrodes at a particular voltage and frequency of a square wave AC signal.

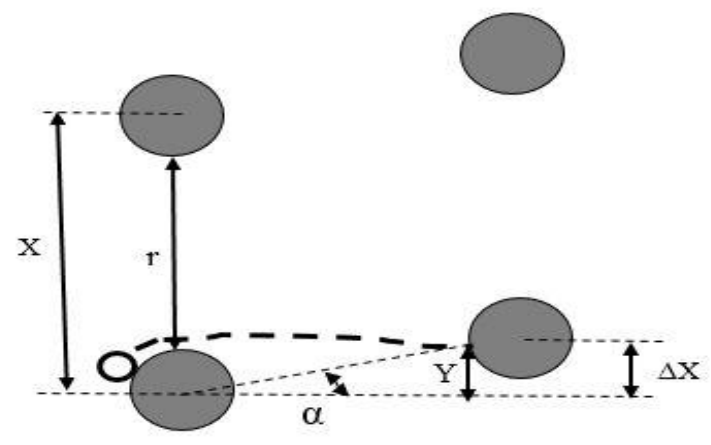

Figure 2 Arrangement of the spot electrodes. 
Working equation for deterministic lateral displacement is as follows:

$Y=(\pi-\alpha) r_{e}+\frac{(X-r)}{2} \sin \alpha(2)$

$r_{c}^{*}=\frac{\Delta X-\frac{X-r}{2}}{(\pi-\alpha)} \sin \alpha(3)$

Where $\mathrm{Y}$ is the lateral displacement of the cell along the flow path. If $\mathrm{Y}>\Delta \mathrm{X}$ then the cell would follow thezigzag path between the electrodes and if $Y<\Delta X$ then the cell would follow the displacement mode. Now $r_{e}$ is the diameter of the cell which decides the value of $Y$ i.e. cells with smaller diameter will flow in zigzag mode and cells with larger diameter will flow in deterministic lateral displacement mode. $r_{c}$ depends on the applied voltage on the electrodes. $r_{\mathrm{c}}{ }^{*}$ is the critical diameter of the cell i.e. if the celldiameteris less than $r_{\mathrm{c}}{ }^{*}$ then the cell will flow in zigzag mode andifitisgreater than $r_{c}{ }_{c}$ then it would flow in deterministic lateral displacement mode. This kind of displacement is called deterministic displacement because the lateral displacement $Y$ of the cell can be determined by the formula equation (2)[2].

The working equation for DEP force is:

$F_{D E P}=2 \pi R^{3} \varepsilon_{m} R E\left(F_{C M}\right) \nabla E_{R M S}^{2}(4)$

$F_{C M}=\frac{\left(\varepsilon_{p}^{*}-\varepsilon_{m}^{*}\right)}{\left(\varepsilon_{p}^{*}-2 \varepsilon_{m}^{*}\right)}(5)$

$\varepsilon_{m}^{*}=\varepsilon_{m}-\frac{\mathrm{j} \sigma_{m}}{\omega}(6)$

$\varepsilon_{p}^{*}=\varepsilon_{p}-\frac{\mathrm{j} \sigma_{p}}{\omega}(7)$

Where, $\nabla \mathrm{E}_{\mathrm{RMS}}$ is the non-uniformity factor of the applied electric field i.e. the rms value of the non-uniform electric gradient between the electrodes, $\mathrm{F}_{\mathrm{CM}}$ is the Clausius-Mosotti factor which depends on the complex permittivity of the medium and the cell which are respectively $\varepsilon_{\mathrm{m}}{ }^{*}$ and $\varepsilon_{\mathrm{p}}{ }^{*}$. In this case complex permittivity has taken in account because the conductivity and the angular frequency of the applied electric field effects the electric permittivity of the cell and the medium as in the equation 6 and 7. Now the complex permittivity effects the $\mathrm{F}_{\mathrm{CM}}$. In case of this device the particles should get repelled by the electric pillars hence we need to get a negative DEP force to achieve the required repelling force.To create a negative DEP force the $\mathrm{F}_{\mathrm{CM}}$ should be negative because it decides the direction of the force with respect to the electric pillars [6].

There is a phenomenon called $\beta$ dispersion in which the cell accepts only the high frequency signals in range of $\mathrm{kHz}$ to few $\mathrm{MHz}$ to pass through it and disperses the high range of frequencies. So the angular frequency should be high enough, so that the cell would allow the high frequency signal to pass through it and the cells would get effected by the electric field.

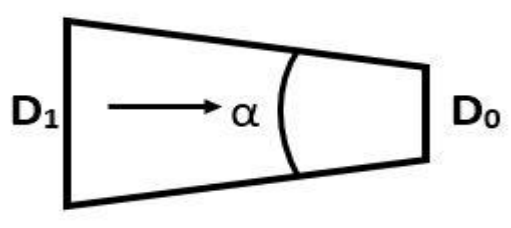

Figure3Nozzle structure.

\subsection{Micro Nozzle Unit}

This unit is a micro fabricated nozzle. Which increases the flow rate of the fluid cell mixture. In case of a nozzle the flow resistance $\zeta_{\mathrm{n}}$ is responsible for the increase in flow rate. $\zeta_{\mathrm{n}}$ can be calculated by the equation 8 (as the Reynolds's number is very low ):

$$
\begin{aligned}
& \zeta_{n}=\frac{A_{n}}{R_{e}}(8) \\
& R_{e}=\frac{\left(v_{o} D_{o}\right)}{v}(9) \\
& A_{n}=\frac{19}{\left\{n_{o}^{0.5}(\tan \alpha)^{0.75}\right\}}
\end{aligned}
$$

Where $R_{e}$ is the Reynolds's number of the flow, $v$ is the kinematic viscosity and $n_{o}=\left(D_{1}{ }^{2} / D_{o}{ }^{2}\right)$. As the fluid gets resistance in its path of flow the pressure starts to drop and according to the law of conservation of energy 
the flow rate of the fluid increases to compensate the loss of energy [5]. The increase of flow rate is required to get faster response from the device.

The nozzle section also contains alternate positive and ground electrodes at its two sides. These electrodes are used for focusing the flow of the cells.

\subsection{Cell Counting Unit}

This unit uses aperture impedance method. In this method there is an aperture and there are two electrodes at both sides of that aperture. The configuration has been shown in figure 4 . The two electrodes are connected through the liquid medium. An AC voltage is applied to the electrodes. Whenever there is a cell in between them there is a change of resistance in the circuit $(\Delta \mathrm{R})$.

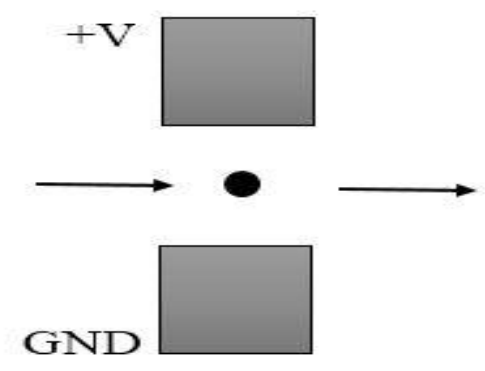

Figure 4 Electrode over the channel with a cell in between them in flowing medium.

The change in resistance is represented by:

$\Delta R=\frac{\rho}{A^{2}} V_{p}(11)$

Where $A$ is the cross sectional area of the channel, $\rho$ is the specific resistance of the medium, $V_{p}$ is the volume of the cell. A and $\rho$ remains constant hence the magnitude of $\Delta R$ depends on $V_{p}[3]$.

\section{Device Simulation Result}

Each unit of the device has been simulated with COMSOL Multiphysics 4.3a.

\subsection{Micro Mixer Unit}

This unit has been simulated with laminar flow domain. Here velocity for blood and PBS has been used $1.1 \mu \mathrm{m} / \mathrm{sec}$ and $2.1 \mu \mathrm{m} / \mathrm{sec}$ respectively. As it results in a uniform flow rate and proper mixing of blood and PBS. The resulting velocity of the diluted blood is $10.61 \mu \mathrm{m} / \mathrm{sec}$.

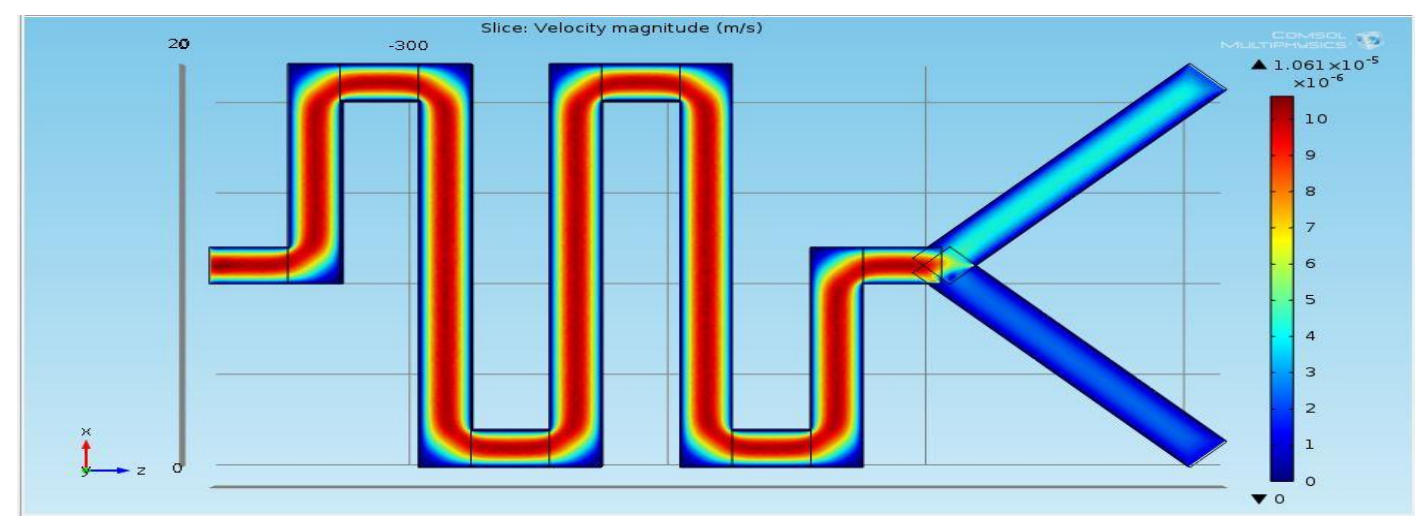

(a) 


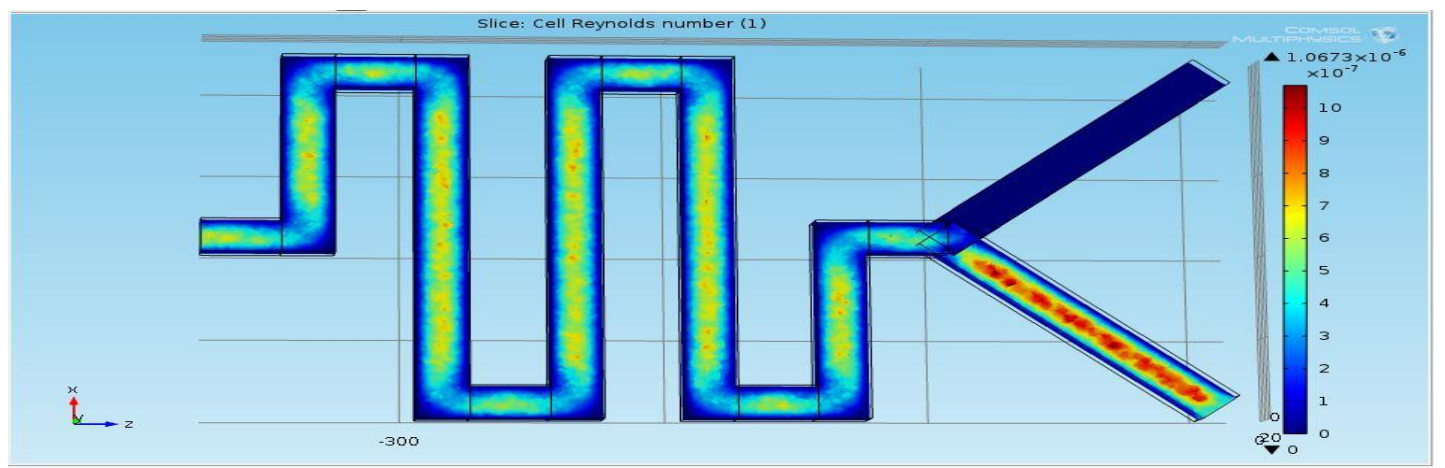

(b)

Figure 5 (a) Simulation of flow velocity in micro-mixer, (b) Simulation of change in Reynolds's number along the channel.

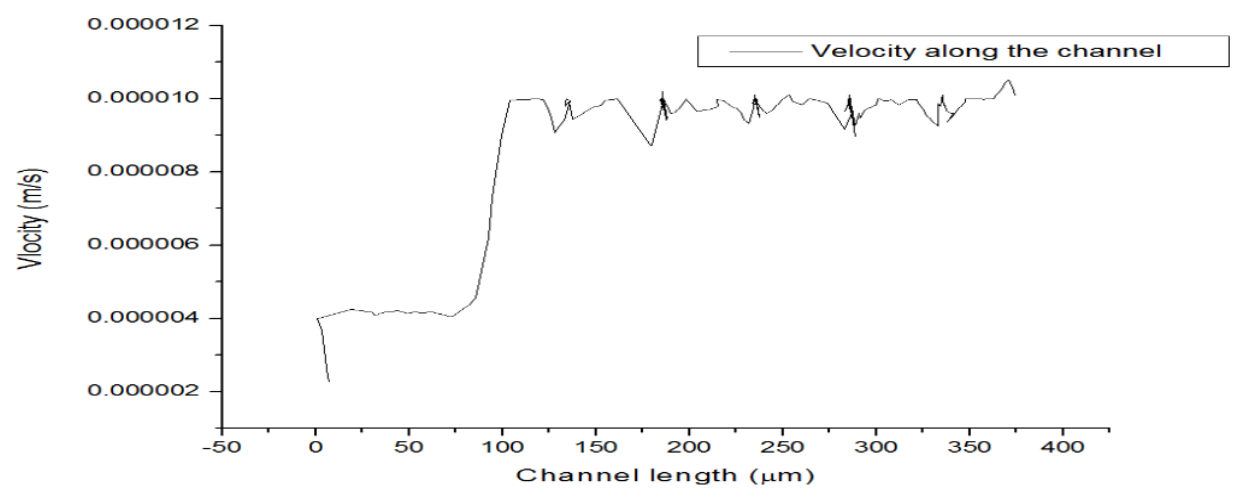

\subsection{Cell Separation Unit}

Figure 6 Flow velocity along the channel of micro-mixer. laminar flow.

The separation unit has been simulated by using domains like electric current, particle tracing and

In electric current domain the alternate electrodes in a row has been assigned as terminals with a voltage signal of $20 \mathrm{~V}$ and other electrodes as ground. The analysis has been done in frequency analysis study with $20 \mathrm{kHz}$ frequency square wave signal to get an $\mathrm{AC}$ signal at the electrodes.

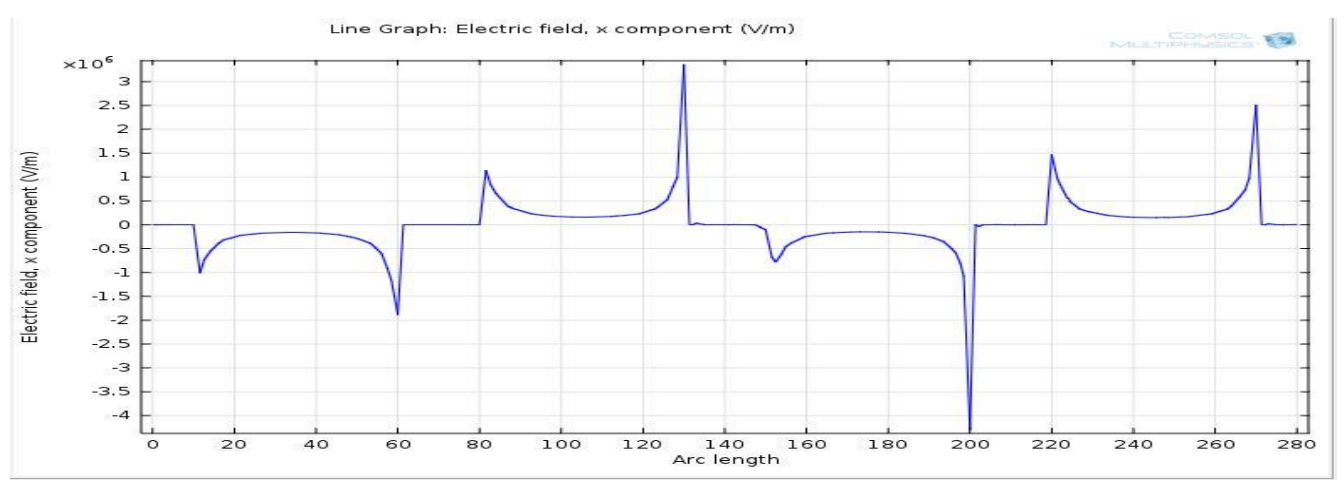

(a) 


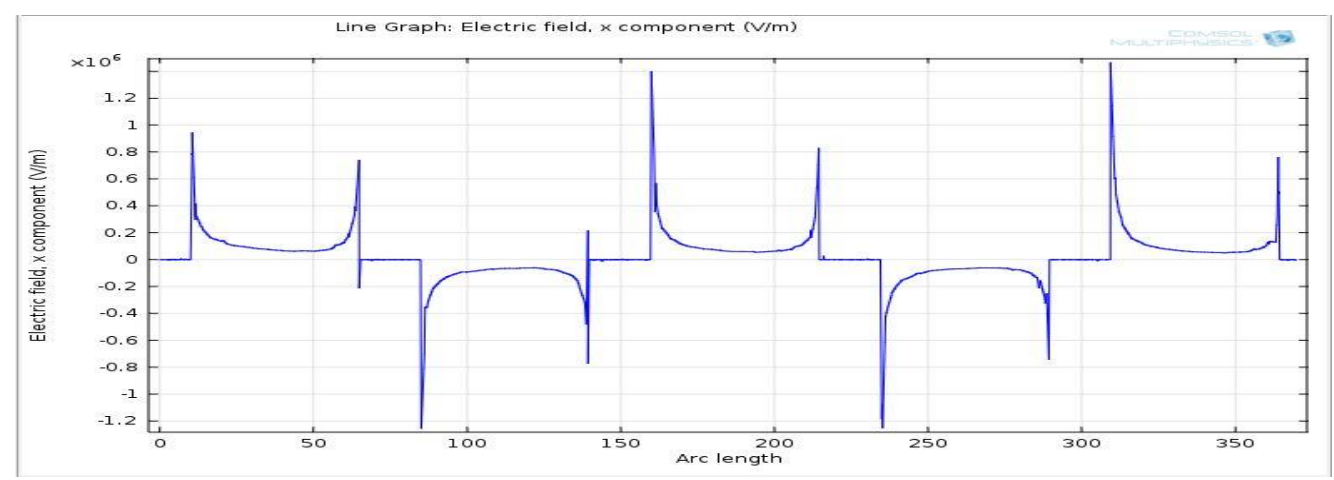

(b)

Figure 7 (a) Electric field along a row of the electrode arrangement. (b) Electric field along a column of the electrode arrangement.

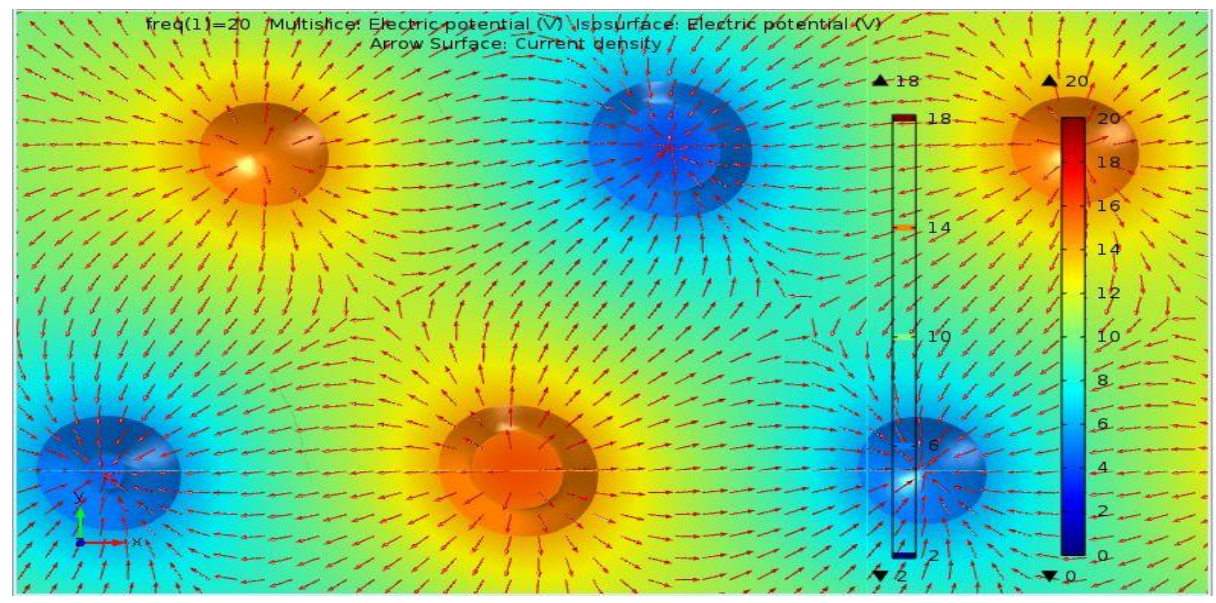

Figure 8 Simulated electrode arrangement in the device with arrows indicating the direction of the electric field.

In particle tracing domain the particles has been used to represent blood cells. In laminar flow domain an inlet flow of $10.61 \mu \mathrm{m} / \mathrm{sec}$ has been used for the diluted blood flow as in this range of velocity the cell gets the proper separation time. In case of higher velocity the cells just gets washed off from the surface.

At $20 \mathrm{~V}$ the electric field covers the electrode and increases the diameter of the electrodes. So that is how we can change the diameter of the electrodes to get the right value of $Y$ which would decide the mode of flow of the cells along the channel. With no electric field applied, the value of $Y$ is equal to $\Delta X$ and $r_{c}{ }^{*}=0.2 \mu \mathrm{m}$. In simulation the channel dimensions has been taken as 500 X $500 \mu \mathrm{m}$ (to test the electrodes functionality in case of the actual device the width would be sufficient but the length of the channel must be increased to get the proper separation). The required $\mathrm{F}_{\mathrm{CM}}$ to create the negative DEP force is -0.2353 .

The particle tracing domain analysis simulation results can't be given in this paper because the animation is impossible to represent in a paper.

\subsection{Micro Nozzle Unit}

This unit has been simulated using only the laminar flow domain.

The inlet dimension of the nozzle is $60 \times 40 \mu \mathrm{m}$ and the outlet dimension is $30 \times 20 \mu \mathrm{m}$. The inlet flow velocity of the nozzle is $0.41134 \times 10^{-6} \mathrm{~m} / \mathrm{s}$ and outlet flow velocity is $3.45 \times 10^{-6} \mathrm{~m} / \mathrm{s}$. This arrangement shows a pressure drop of $0.0152 \mathrm{~Pa}$. The flow resistance co-efficient is 0.226 . 


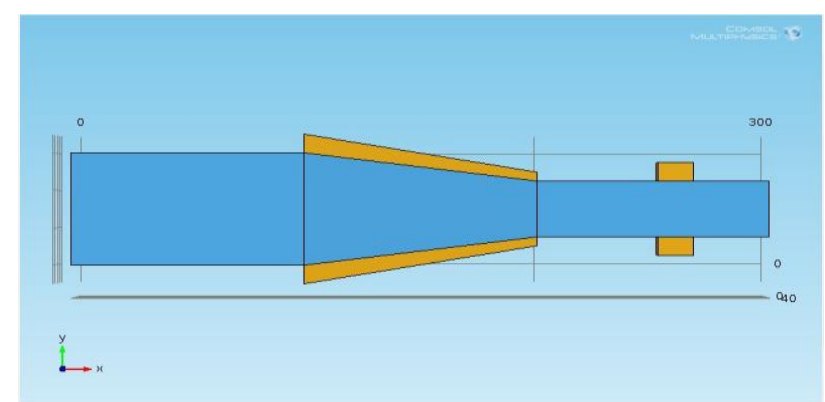

Figure 9Simulation of the cell focusing electrodes at the micro nozzle and the counter electrodes.

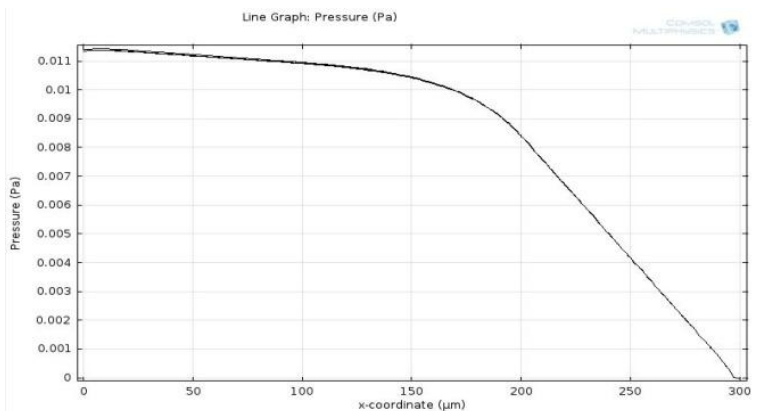

(a)

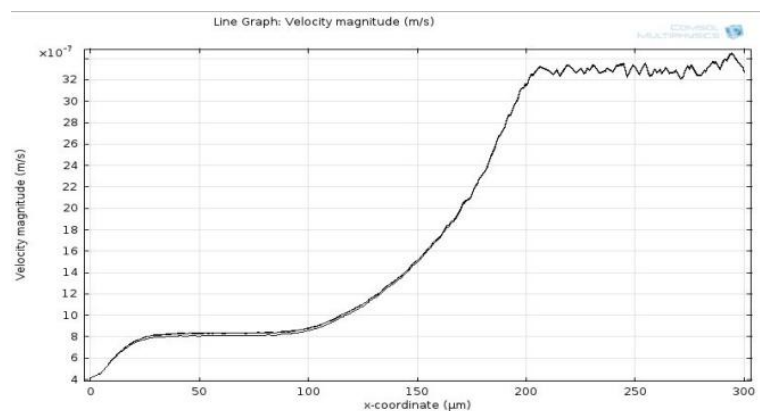

(b)

Figure10 (a) Pressure drop along the channel, (b) Flow velocity magnitude along the micro nozzle.

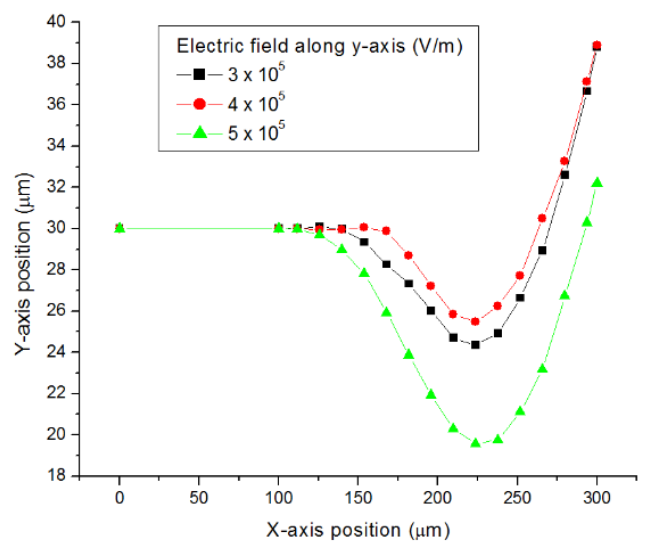

(a)

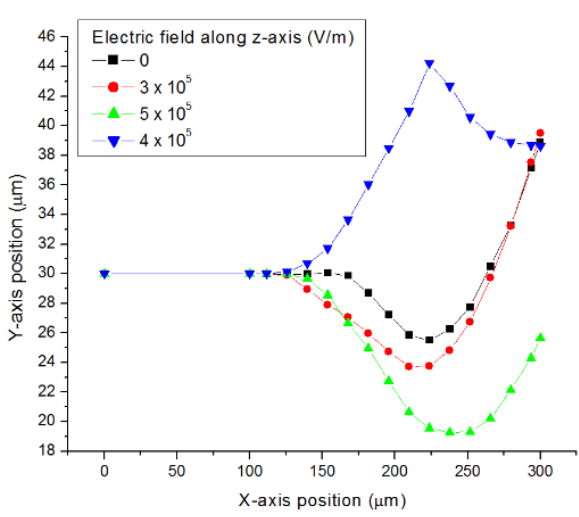

(b)

Figure 11 (a)Trajectory of the cell along the channel for different electric field along y-axis,(b) Trajectory of cell along the channel when electric field along y-axis is $4 \times 10^{5} \mathrm{~V} / \mathrm{m}$ and electric field along $\mathrm{z}$-axis has been varied.

From Fig. 11 (a) it is evident that when $4 \times 10^{5} \mathrm{~V} / \mathrm{m}$ electric field is applied to the focusing electrodes along yaxis, cells are following a more linear path than the other applied electric fields. So 7V square wave AC voltage is applied to the focusing electrodes applying force along y-axis. Form figure 11 (b) it can be seen that when another set of focusing electrode has been used to apply an electric field along z-axis the particles becomes more non-linear than without this focusing electrode. But as the test has been done on simulated cells not on the actual biological cell. Hence it is expected that in case of the actual biological cell the focusing electrodes at $\mathrm{z}$-axis would be required and an electric field of $3 \times 10^{5} \mathrm{~V} / \mathrm{m}$ should be applied to the electrodes. The two sets of focusing electrodes would act as a lens to focus the cells.

Hence the cell focusing electrodes are excited by square wave AC signal of $7 \mathrm{~V}$ and $20 \mathrm{kHz}$ frequency to keep the cell flow in a linear path and most preferably along the central line of the nozzle. 


\subsection{Cell CountingUnit}

This unit has been simulated using laminar flow, electric current and particle tracing physics.

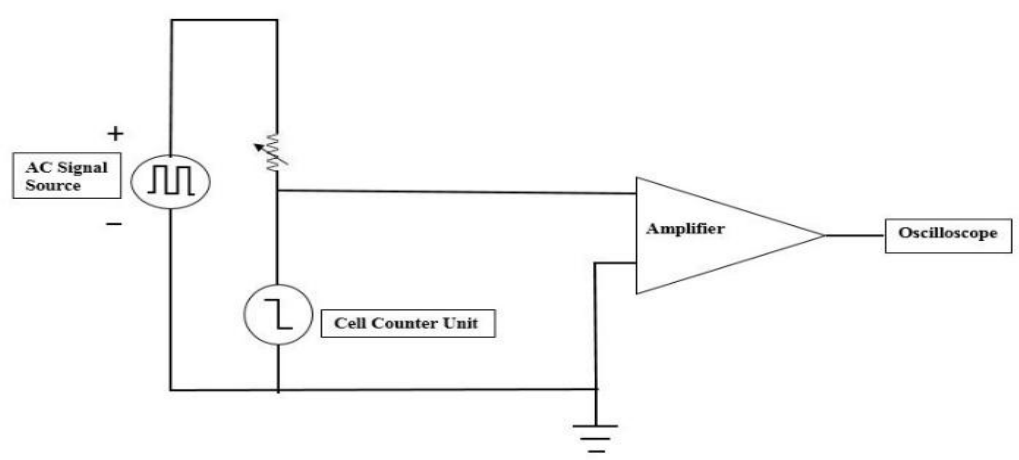

Figure 12 Cell counter circuit.

The circuit used for sensing the change in resistance has been given in the Fig. 12. The cell counter unit is connected in a voltage divider circuit and the output of that voltage divider is connected with an amplifier, so that if any resistance change occurs in the counter unit the amplifier would give a pulse equivalent to the resistance change.

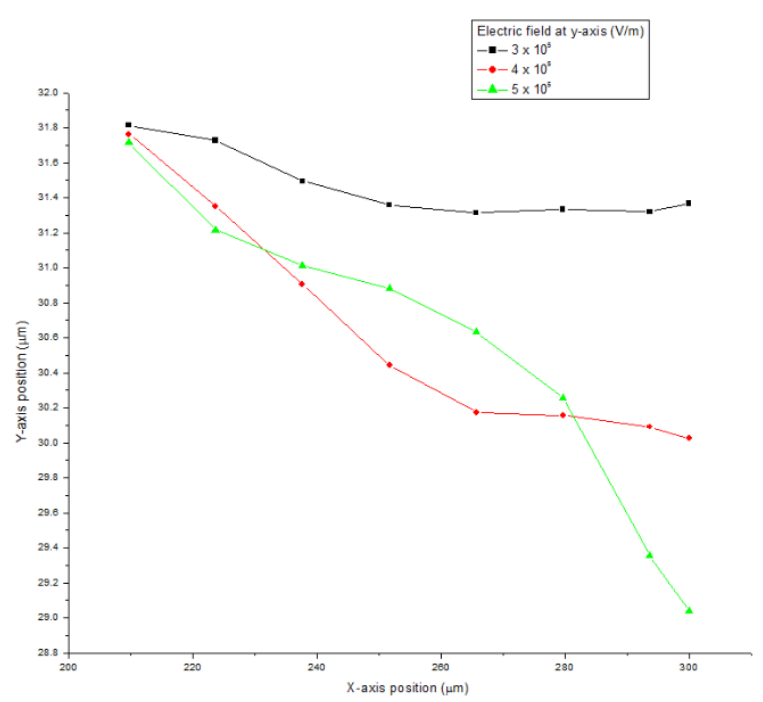

Figure 13 Trajectory of the cell along the channel after the micro-nozzle unit for different electric filed applied to the y-axis.

From Fig. 13 it can be seen that the cell motion along the channel is linear when the applied electric field is $3 \mathrm{x}$ $10^{5} \mathrm{~V} / \mathrm{m}$. But at $4 \times 10^{5} \mathrm{~V} / \mathrm{m}$ as the cell comes nearer to the counter electrodes, it gets more focused at the center of the channel width as desired. Hence $4 \times 10^{5} \mathrm{~V} / \mathrm{m}$ electric field at $\mathrm{y}$-axis is appropriate for the counter electrodes.

The inlet flow of this unit is as same as the outlet of the micro nozzle. The electrodes used for sensing the change in resistance are excited by $20 \mathrm{kHz}$ square wave $\mathrm{AC}$ signal as with a voltage of $7 \mathrm{~V}$.

From figure 14 it can be seen that when cell is not present in between the electrodes the voltage at the middle of the channel is much higher than the times when the cell is present. As the cell is an obstraction to the electric field, the voltage when cell is present ranges from 0 to $0.5 \mathrm{~V}$. This change in voltage can be detected by the circuit and the out put can be given accordingly. Every time a cell passes through the gapbetween the electrodes this changeoccures and a pulse is generated. Thus it can detect the cells and the number of pulses give the count of wbc. 


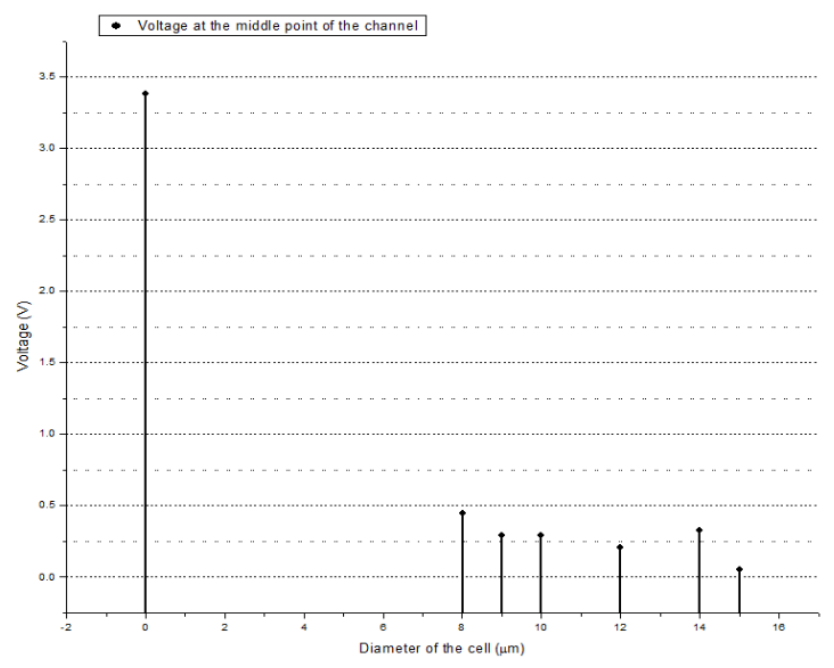

Figure 14 Change in voltage in between the electrodes due to prescence of cell of different sizes.

\section{Conclusion}

The MEMS based device has been simulated using COMSOL Multiphysics 4.3a. The results were satisfactory. To get proper results physical properties of human blood and PBS has been used for the simulated fluids. The cell separation unit has been simulated with the proper voltage excitation. The micro nozzle part is increasing the flow velocity as desirable and its cell focusing electrodes are allowing the flow of cell to be linearized. But due to lack of availability of fabrication facility, the device is still in design phase. Hence the results have been derived from the simulation rather than from the fabricated device. The results presented in the paper are only simulation results and practical results are unavailable. The circuit to count the Leukocytes has been designed and would be implemented practically and calibrated only after the device gets fabricated. Also the length of the separation channel can be decided only after fabrication and testing of the fabricated device. It is expected that the fabrication facility would be arranged in future and this device would get fabricated as soon as possible. It is believed that this device would help doctors as well as common people to diagnose their Leukocyte related diseases quicker and with more ease.

[1] SiyangZhmg, Raylene Yung, Yu-Chong Tail, Harvey Kasdan“Determinstic lateral displacement mems device for continuous blood cellseparation", Caltech Micromachining Lab, California Institute of Technology, USA,StanfordUniversity, USAInsDiagnostics, International Remote Imaging Systems, Inc., USA, 8732-5/05 IEEE851-854 2007

[2] Young-Ho Cho, "Bio-Inspired MEMS Devices forElectrical Cell Separation and Mechanical Cell Characterization", Digital Nanolocomotion Center, Korea Advanced Institute of Science and Technology (KAIST) 373-1 Guseong-dong, Yuseong-gu, Daejeon 305-701 Republic ofKorea 978-1-4244-1858-9/07 IEEE 514 - 5182007

[3] Daisuke Satakea,, Hiroyuki Ebia, NarihiroOkub, KoichiroMatsudaa, HidekuniTakaoc, MitsuakiAshikic, Makoto Ishidac, “A sensor for blood cell counter using MEMS technology", Fundamental Technology ResearchDepartment, HORIBA Ltd., 2 Miyanohigashi, Kisshoin,Minami-ku, Kyoto 601-8510, Japan,Medical Electronic Systems, HORIBA Ltd., 2 Miyanohigashi, Kisshoin, Minami-ku, Kyoto 601-8510, Japan,Department of Electrical and Electronic Engineering, Toyohashi University of Technology, 1-1, Hibarigaoka, Tempaku-cho,Toyohashi 441-8580, Japan.Sensors and Actuators B 83 77-81 2002

[4] M. Korampally, J. D. Benson, Y. Wu, 1 J. K. Critser, M. Almasri, "MEMS based Coulter counter for cell sizing”,Department of Electricaland Computer Engineering, and Department of Veterinary,Pathobiology, University of Missouri, Columbia, Missouri, 65201, USA. Proc. of SPIE Vol. 6886, 68860A, 0277-786X/08/\$18 · doi: 10.1117/12.763741 2008 1-7

[5] X.N.Jiang, Y.Zhou, Y.Li, Y.Yang, X.Y.Huang, and C.Y.Liu. "Experiments and analysis for micronozzle/diffuser flow and microvalveless pumps", School of MPE, NanyangTechnological University Singapore639798,Dept. of Precision Instruments and Mechanology, TsinghuaUniversity, Beijing, 100084,China.0-7803-3829-4/97 1997 IEEE 369 - 372

[6] S. Burgarella, M. Bianchessiand M. De Fazio " Numerical modeling of dielectrophoretic forces acting uponbiological cells in silicon lab-on-chip devices", STMicroelectronics, Advanced System Technology, R\&I e-Health ,Corresponding author: STMicroelectronics, Centro Dir. Colleoni, Pal. Dialettica, Via Cardano 1, 20041AgrateBrianza (MI), Italy.Excerpt from the Proceedings of the COMSOL Users Conference Grenoble 2007 